\title{
Guest Editorial: Intelligent Decision Making and Consensus Under Uncertainty in Inconsistent and Dynamic Environments
}

In today's Information and Knowledge Society, thanks to the new Information and Communication Technologies, we have many more information resources that allow both individuals and large entities to make decisions anywhere, anytime and with more knowledge. However, this greater amount of available information is not always easily processable, consistent, understandable, accurate and complete. This makes the task of making decisions more complex. Intelligent Decision Making Support Systems (IDMSS) and consensus models, which involve the design of mathematical models for modeling decision making problems, the use of different Information Technologies (such as the Web, Web 2.0, Mobile Internet and social networks) and Artificial Intelligence tools (such as Computational Intelligence tools), are of great importance to deal with such complexity. To address the decision making challenge in many risky and complex frameworks, IDMSS are emerging from the Computer Science field to aid experts, users and/or large entities to make correct decisions and provide robust automation to solution to problems. On the other hand, the development of formal mathematical models to support experts in making decisions is of great importance to assure the validity of the actions derived from a decision outcome. This is of special relevance in decision contexts where the information on the problem at hand is not amenable to be modelled in a quantitative and precise way. Another issue to be addressed is that of inconsistency of information and the dynamic nature of the decision making process itself. This type of decision-making is now being described as decision-making under uncertainty in inconsistent and dynamic environments.

The special issue has brought together researchers to report their latest and best results regarding the development of IDMSS and consensus models under uncertainty in inconsistent and dynamic decision environments. We have received seventy contributions from authors working on this topic 
from all parts the world. After a rigorous anonymous peer review process, without bias in the referee process when we received guest editors' contributions, twenty high quality papers were accepted to be included in this special issue, which have been grouped into three groups. The first one includes eight papers presenting new consensus models for group decision making problems under uncertainty in inconsistent and/or dynamic decision environments. The second one includes six papers presenting novel theoretical models of IDMSS under uncertainty in inconsistent and/or dynamic decision environments. The third one consists of six real-life applications of IDMSS under uncertainty in inconsistent and/or dynamic decision environments.

The first group of submissions begins with a paper by Yucheng Dong, Quanbo Zha, Hengjie Zhang, Gang Kou, Hamido Fujita, Francisco Chiclana and Enrique Herrera-Viedma, "Consensus Reaching in Social Network Group Decision Making: Research Paradigms and Challenges", that reviews existent consensus models in social network group decision making (SNGDM) by identifying two paradigms: (i) the consensus paradigm based on trust relationships, and (ii) the consensus paradigm based on opinion evolution. Additionally, major research challenges to be addressed in the future are also provided. The paper entitled "The consensus of probabilistic uncertain linguistic preference relations and the application on the virtual reality industry", by Xie Wanying, Ren Zhiliang, Xu Zeshui, and Wang Hai, presents a new consensus model for group decision making under probabilistic uncertain linguistic preference relations and its application in the selection of virtual reality companies. Zhang Hengjie, Iván Palomares, Yucheng Dong and Weiwei Wang propose a novel consensus model based on social network analysis to deal with experts that exhibit non-cooperative behaviors in their paper titled "Managing non-cooperative behaviors in consensus-based multiple attribute group decision making: An approach based on social network analysis", with its main novelty being a trust propagation and aggregation mechanism to yield experts' weights from the social trust network. The paper "Ranking objects from group decision making with interval-valued hesitant fuzzy preference relations in view of additive consistency and consensus", by Jie Tang and Fanyong Meng, includes a group decision making model based on interval-valued hesitant fuzzy preference relations and different aspects related with the additive consistency property, its use to manage incomplete preferences and the design of the consensus processes in such decision context. The next paper by Wenqi Liu, Hengjie Zhang, Xia Chen and Shui Yu, "Managing consensus and self-confidence in multiplicative preference relations in 
group decision making", describes a new consensus model for group decision making problems with self-confident multiplicative preference relations that introduces a new two-step feedback adjustment mechanism to assist the decision makers to improve their consensus level. The paper titled "A two-fold feedback mechanism to support consensus-reaching in social network group decision-making", by Zhang-peng Tian, Ru-xin Nie, Jian-qiang Wang and Hong-yu Zhang, defines a new consensus model for group decision making in social network that operates according to two feedback mechanisms: one providing recommendations to the experts to change their judgements and the other one modifying the experts' weights as an incentive or penalty to force them to accept the received suggestions. The decision framework allows to compute the experts' weights by means of both interval-valued dual trust propagation operators and knowledge based induced OWA operators. Zhang Bowen, Liang Haiming, Gao Yuan and Zhang Guiqing, in their paper "The optimization-based aggregation and consensus with minimum-cost in group decision making under incomplete linguistic distribution context", define a novel consensus model for group decision making with incomplete linguistic distribution assessments based on a consensus-oriented aggregation approach to derive a collective opinion with maximum consensus, and further developed a minimum-cost consensus model with variable unit consensus cost. In the next paper "Geo-uninorm Consistency Control Module for Preference Similarity Network Hierarchical Clustering Based Consensus Model", Nor Hanimah Kamis, Francisco Chiclana, and Jeremy Levesley present a new consensus approach to solve group decision making problems with reciprocal preference relations that implements rationality criteria of consistency based on the transitivity property with the following twofold aim prior to finding the final decision solution: (A) to develop a consistency control module to provide personalized consistency feedback to inconsistent experts; and (B) to design a consistent preference network clustering based consensus measure based on an undirected weighted consistent preference similarity network structure with undirected complete links, which using the concept of structural equivalence will allow one to (i) cluster the experts; and (ii) measure their consensus status.

The second group of submissions includes a first paper titled "The stratified multi-criteria decision-making method", by Mehdi Rajabi Asadabadi, that presents a new model of IDMSS that implements a multiple criteria decision making method based on the concept of stratification that defines new stratified and dynamic decision frameworks where possible fluctuations in 
the criteria weightings are supported. The next paper "IFS/ER-based largescale multiattribute group decision-making method by considering expert knowledge structure", by Yuan-Wei Dua, Ning Yang and Jing Ning, introduces a new model of IDMSS that uses a large-scale multiattribute group decision-making model under interval-valued intuitionistic fuzzy preferences that takes into account the expert knowledge structure, with an information extraction mechanism that provides three kinds of inference ways including singleton attribute inference, local integral inference and global integral inference being introduced to ensure the assessments made by each expert with interval-valued intuitionistic fuzzy values to be valid. In the paper "Insights into tolerability constraints in multi-criteria decision making: Description and modeling", Xiaoyang Yao, Jianping Li, Xiaolei Sun and Dengsheng Wu present a new model of IDMSS for tolerability constraints in the context of non-independent criteria. In this IDMSS global tolerability constraints on the overall decision making process and local constraints on a single criterion are considered at the same time. Furthermore, mandatory/sufficient requirements are regarded as different aspects of tolerability constraints. Sylvain Kubler, William Derigent, Alexandre Voisin, Jérérmy Robert, Yves LeTraon and Enrique Herrera-Viedma in their paper "Measuring Inconsistency and Deriving Priorities from Fuzzy Pairwise Comparison Matrices using the Knowledge-based Consistency Index" introduce a new model of IDMSS based on a fuzzy AHP that introduces a new knowledge-based consistency index for fuzzy comparison matrices and provides an optimal solution to multicriteria decision making problems according to the decision maker's viewpoint and expertise. In the paper "Related families-based attribute reduction of dynamic covering decision information systems", Guangming Lang, Mingjie Cai, Hamido Fujita and Qimei Xiao show a new model of IDMSS that uses related family-based approaches and incremental algorithms for attribute reduction of dynamic covering decision information systems in terms of attribute arriving and leaving. This decision model is developed using granular computing and rought sets. The last paper of this group by Zaiwu Gong, Ning Zhang and Francisco Chiclana, "The Optimization Ordering Model for Intuitionistic Fuzzy Preference Relations with Utility Functions", describes a model of IDMSS to deal with group decision making problems under intuitionistic fuzzy preference relations. The model provides an optimal ranking of alternative using utility functions.

The last group of submissions starts with the paper "Risk evaluation by FMEA of super-critical water gasification system using multi-granular lin- 
guistic distribution assessment", by Nie Ru-xin, Tian Zhang-peng, Wang Xiao-kang, Wang Jian-qiang and Wang Tie-li, in which an IDMSS based on multi-granular linguistic modelling is applied in risk evaluation by failure mode and effects analysis in super-critical water gasification systems to ensure the elimination and recovery of pollution produced by sewage sludge treatment. Ying Yang, Dong-Ling Xu, Jian-Bo Yang and Yu-Wang Chen, in their paper "An evidential reasoning-based decision support system for handling customer complaints in mobile telecommunications", define a novel IDMSS for handling customer complaints by developing an evidential reasoning rule-based classifier as the core component of the system to classify customer complaints with uncertain information, which is applied in a telecommunication company. The paper "Identifying items for moderation in a peer assessment framework", by S. James, E. Lanham, V. Mak-Hau, L.Pan, T.Wilkin and G. Wood-Bradley, describes a new IDMSS to aid in peer assessment frameworks that can be very useful in education contexts to greatly reduce the workload of educators. The authors firstly define several indices that when combined can be used to estimate the reliability of peer markers and secondly, by considering the consensus of scores received by peers, a subset of peers whose results should be flagged for moderation is identified. In the paper "A Multi-Criteria Evaluation Approach in Navigation Technique for Micro-jet for Damage \& Need Assessment in Disaster Response Scenarios", Tamal Mondal, Indrajit Bhattacharya, Prithviraj Pramanik, Naiwrita Boral, Jaydeep Roy, Subhanjan Saha and Sujoy Saha propose a new IDMSS to deal with spatial decision problems. This system works as a navigation technology to find the best navigation path according to parameters such as wind speed, wind direction, remaining flight time, the distance between the neighboring Shelter Points and the volume of data to be transferred. Xue Min, Fu Chao, Feng Nan-Ping, Lu Guang-Yan, Chang Wen-Jun and Yang Shan-Lin in their paper "Evaluation of supplier performance of high-speed train based on multi-stage multi-criteria decision-making method" present a new IDMSS based on a multi-stage multi-criteria decision-making method that evaluates the performance of suppliers in the industry of high-speed train manufacturing via an evidential reasoning approach modelled by belief distributions. The last paper of this group by Jose Ignacio Peláez, Francisco E. Cabrera and Luis G. Vargas, "Estimating the Importance of Consumer Purchasing Criteria in Digital Ecosystems", define a new IDMSS based on a purchasing-decision prediction models that estimates the importance of the criteria and values of the alternatives by considering just the opinions ex- 
pressed by consumers in digital ecosystems, while using an expert-defined set of criteria. The proposed approach extracts the weights of both criteria and alternatives and determines the implicit synergies within them without the need of additional input. The system uses a Choquet integral for weight and valuation aggregation and determines a purchase ranking.

We believe that this collection of research papers contains new research trends and challenges related to IDMSS and consensus models and that it will contribute researchers to achieve new advances in this research field. Finally, as Guest Editors of this special issue, we would like to thank all the authors for their contributions as well as the anonymous referees for their outstanding cooperation and constructive input.

Enrique Herrera-Viedma

University of Granada, Granada, Spain

viedma@decsai.ugr.es

Francisco Chiclana

De Montfort University, Leicester, UK

University of Granada, Granada, Spain

chiclana@dmu.ac.uk

Yucheng Dong

Sichuan University, Chengdu, China

ycdong@scu.edu.cn

Vincenzo Loia

Salerno University, Salerno, Italy

loia@unisa.it

Gang Kou

Southwestern University of Finance and Economics, Chengdu, China

kougang@swufe.edu.cn

Hamido Fujita

Iwate Prefectural University, Japan

HFujita-799@acm.org 